\title{
The Alexander L. Kielland Disaster Revisited: A Review by an Experienced Welding Engineer of the Catastrophic North Sea Platform Collapse
}

\author{
E. J. France
}

Submitted: 9 July 2019/ Accepted: 14 July 2019/Published online: 24 July 2019

(C) The Author(s) 2019

\begin{abstract}
The objective of this review regarding the catastrophic failure of an important welded structure, the Alexander L. Kielland platform, was to indicate that the investigation team should have comprised and involved an experienced welding engineer. Lack of involvement of the welding engineer can lead to misinterpretation of the evidence which results in the incorrect identification of the cause of failure. In this case, the Alexander L. Kielland platform, only the mechanism of failure, which was by fatigue, was correctly identified by the investigation team, this review by the author has correctly identified the true causes that lead to catastrophic failure. Derived from identification of the true causes, i.e. a need for more appropriate design criteria and suitable welding protocols, then by the rectification of these causes, we can suppress the vulnerability of the structure to these modes of fatigue failure operating. The result of this review should therefore be welcomed by anyone concerned with design, construction and the health and safety of operation of these types of welded structure.
\end{abstract}

Keywords The Alexander L. Kielland .

Welding metallurgy · Welded structure · Cause of failure

\section{Introduction}

The Alexander L. Kielland rig/platform was fabricated and completed in March 1976; it collapsed on 27 March 1980, whilst in service, due to a fatigue crack propagating from a welded joint, with the loss of 123 lives. The original design

E. J. France $(\bowtie)$

Manchester, UK

e-mail: enquiries@ejfranceconsulting.co.uk of the construction was a standalone drill rig, with associated service facilities, for service in a shallow marine environment. The platform was supported vertically by five tubular columns at the apices of a pentagonal design, each interlinked by robust tubular braces and stabilized upon pontoon bases. The structure entered service and ended up as a "flotel" accommodating several hundred rig personnel. The failure sequence was identified as the loss of one vertical column, resulting in rig destabilization, with tilting of the entire structure leading to inversion and submersion. All the evidence confirmed the mechanism of the initial failure as a fatigue process, sited at a defective weld bead in an inserted hydrophone tube located in a robust brace designated D-6. The fracture characteristics of the principle failure site were subject to extreme scrutiny, and it was the conclusions of all the major reports that a progressive cracking in that brace and the consequent brace separation that led to the ultimate collapse of the entire structure. At this point, the author confines his attention to the causes of the disaster whilst acknowledging with deep regret, the extent of this human tragedy.

\section{Investigation and Commentary}

Historically, large welded structures have, and continue to have, concerns regarding the integrity of the welded joints. All welds are imperfect, yet despite an evolving attention, concern and scrutiny by government, insurance, inspection and regulatory bodies, what real advances have been achieved in reducing the probability of structural failures? Focus on the design, material selection and weld protocols do produce welds with the requested physical structures and mechanical properties, yielding the intended component lifetimes in service. Using the cited failure, however, 
as the targeted example, was the weld completed in a satisfactory manner? The answer is a resounding-No !!!

A brief examination of the fatigue characteristics defines the site of crack initiation at and around the inserted hydrophone tube, in the brace D- 6 . The site of the primary progressive fracture together with the later plastic failures is shown by the circumferential red markings in Fig. 1.

The primary fracture path is defined in Fig. 2 with the inserted hydrophone tube remaining in place beside the drainage port.

Clearly, part of the insert weld remained integral since the components are still rigidly attached after some 3 years of service. The entire surface fracture characteristics were scrutinized to establish, unequivocally, the changing mechanisms of fracture along this path, Fig. 3. The results of this study are conclusive, with two fatigue fracture initiation sites (I and II see Fig. 3), at diametrically opposite points within the overall fracture plane. The cyclic fatigue crack paths were characterized by "clam shell" markings which arise from minor changes in the direction of the crack tip during progressive crack extension. These pathways were approximately $312 \mathrm{~cm}$ (II) and $113 \mathrm{~cm}$ (I) in lengths, prior to the more rapid crack extension by both ductile fibrous tearing and latterly by brittle fracture shown by the chevron markings. In total, the fatigue crack components represented about 53\% of the crack surfaces. Investigators reported the presence of paint layers within the intended weld bead, which confirmed the defective weld condition before the structure entered service. The lifetime of the brace D-6 is significant; it also confirms and identifies that the quality of the steel used in its fabrication was appropriate.

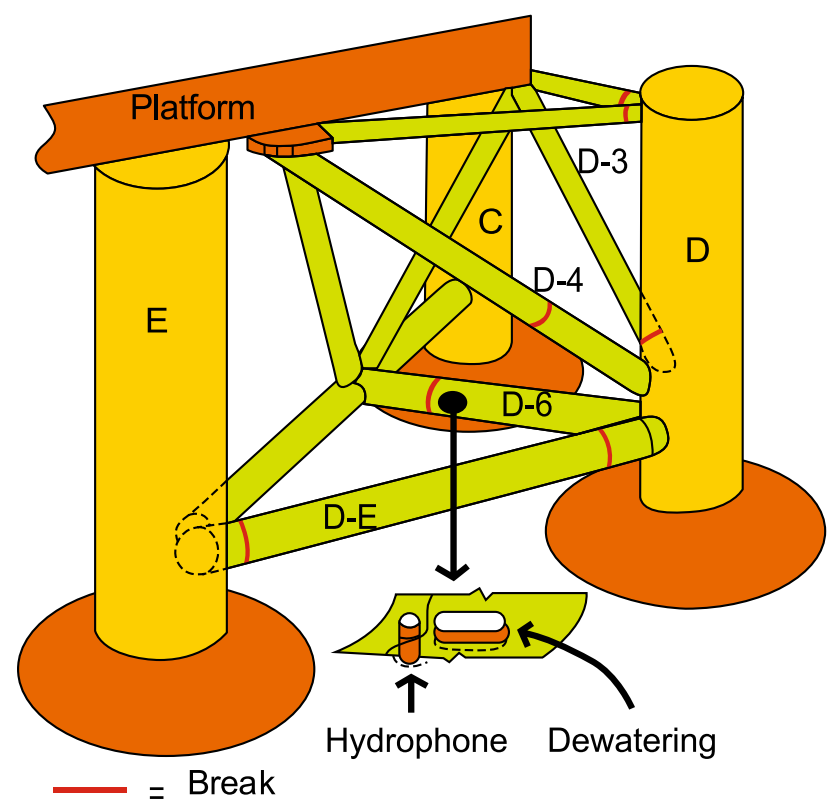

Fig. 1 Position of the fatigue fracture on brace D-6, marked in red
At this point in the review, a welding engineer would demand a detailed study of the weld design and welding protocols. It was and remains a fact that the weld was produced in a zone of designated and low nominal stress level, using materials which met the required chemical and mechanical property specifications. The hydrophone support was inserted and welded into the brace as a "set through, pipe to pipe, double sided fillet, branch weld". The dimensions of the intended joint configuration are shown in Fig. 4.

The welding engineer would regard the joint as a point of difficulty and would not have recommended a simple fillet weld between thick wall components, which involved inserting a large circular hole into the brace, i.e. a cavity of $325 \mathrm{~mm}$ diameter. The weld protocol requested a $6 \mathrm{~mm}$ throat thickness fillet, alternatively expressed as an $8 \mathrm{~mm}$ leg length fillet weld, when estimated by a weld gauge. It was known that the thick-walled brace had been manufactured with both longitudinal and circumferential "full thickness penetration butt welds" and had been inserted into the fabrication as such. Why was the hydrophone insert tube not required to also have full penetration butt welds, perhaps with the reinforcement as indicated in Fig. 5?

The accident report [1].

The incident prompted a post-collapse study of the platform design, construction requirements and the metallurgical examination of the recovered components to ascertain the probable cause(s). The author will confine his comments to the welding criteria and forensic metallurgical evidence contained within the accident report [1]. The fabrication protocols listed all weldment design, weld quality and post-weld inspection criteria. Weld joints were classed under three sections, based predominantly upon the anticipated stress levels in service, viz. high, medium and low, see Fig. 6.

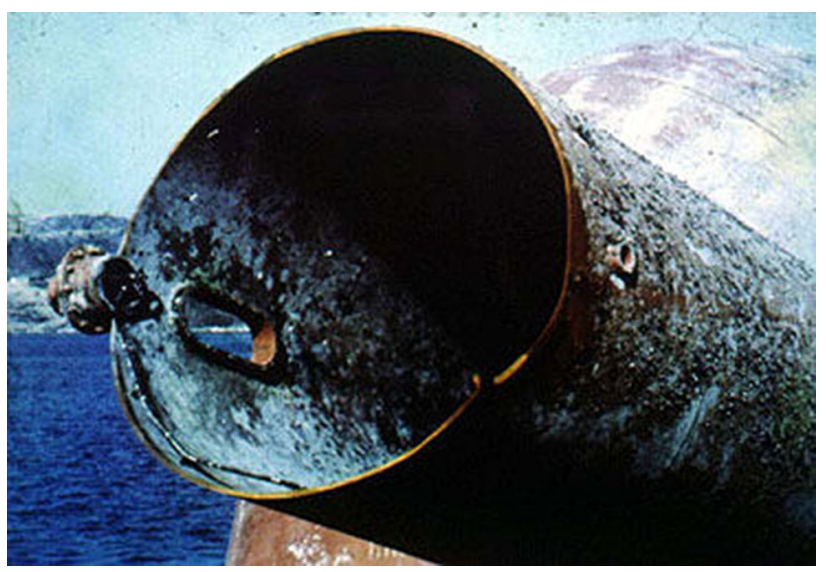

Fig. 2 Fatigue crack propagated part way round the insert and then the brace 

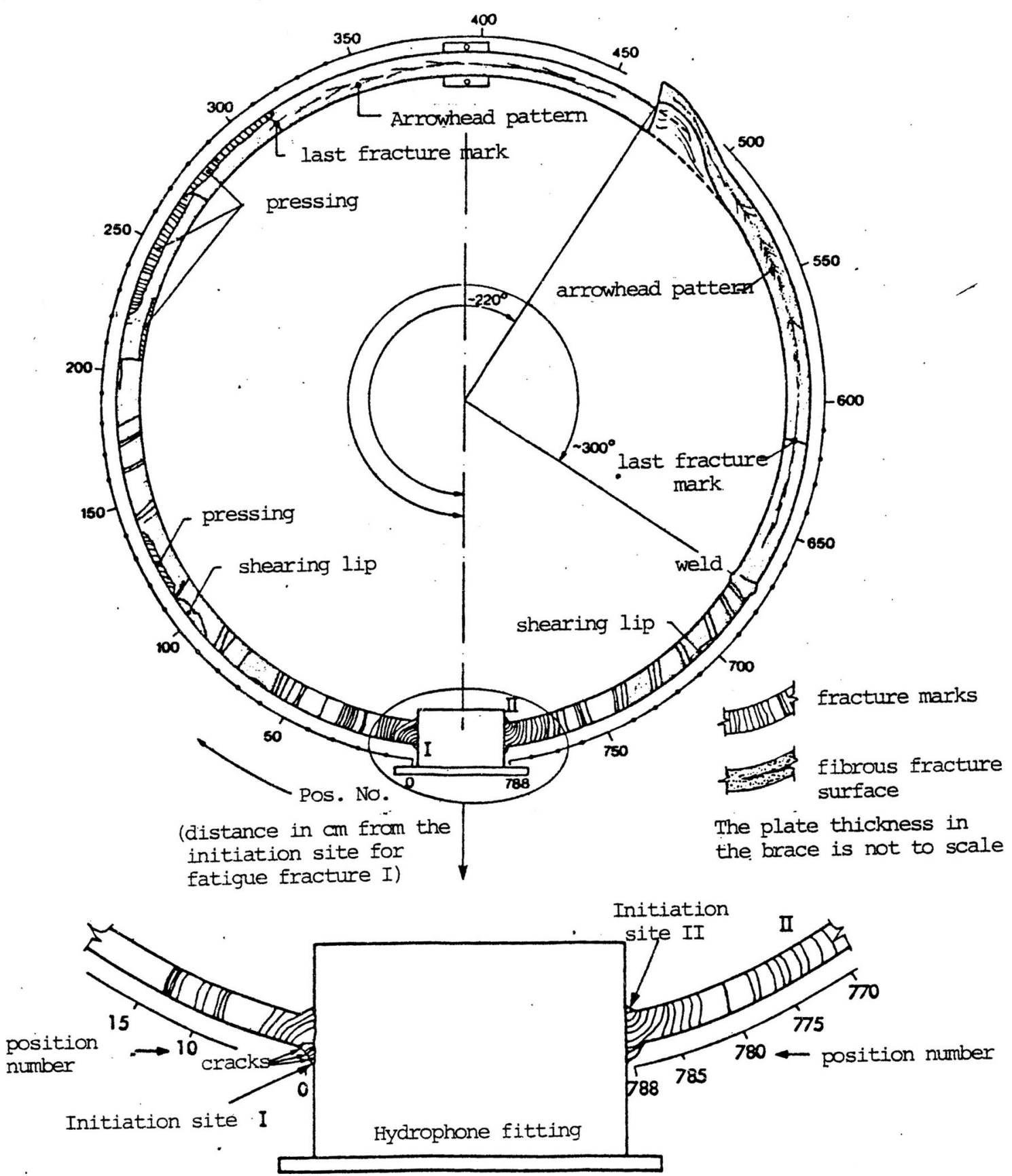

Fig. 3 Schematic representation of the fracture surface around the hydrophone tube taken from the accident report[ 1, p 338]

Class 3 referred in particular to the connection of hydrophone fittings in braces.

It was unclear where the use of qualified weld procedures and welders were mandatory, but they were certainly expected for Class 1 and Class 2. Class 3 types, as deemed to be employed in the hydrophone tubular insert, would therefore only require a relatively low strength weld, e.g. a small fillet weld, and would be inspected by the welder alone, unless inspection by Dye Penetrant/Magnetic Particle technique was designated. The failure mechanism was studied to evaluate both the weld integrity and the potential sources of associated weld defects, using a macrostructural examination technique applied to the recovered brace component. Now, see Fig. 7, which is taken from page 342, 


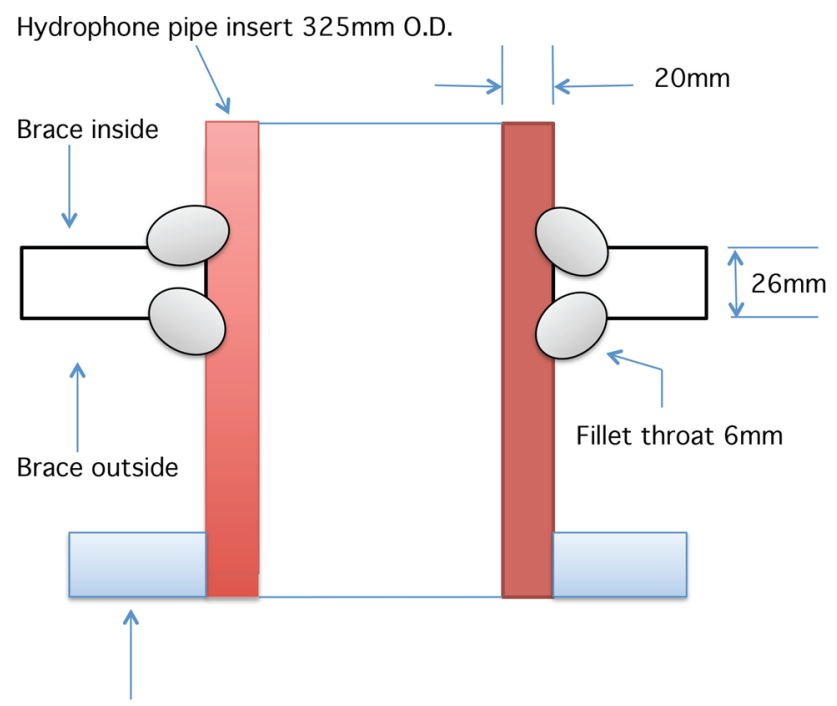

Slip on flange

Fig. 4 Dimensions of hydrophone insert joint

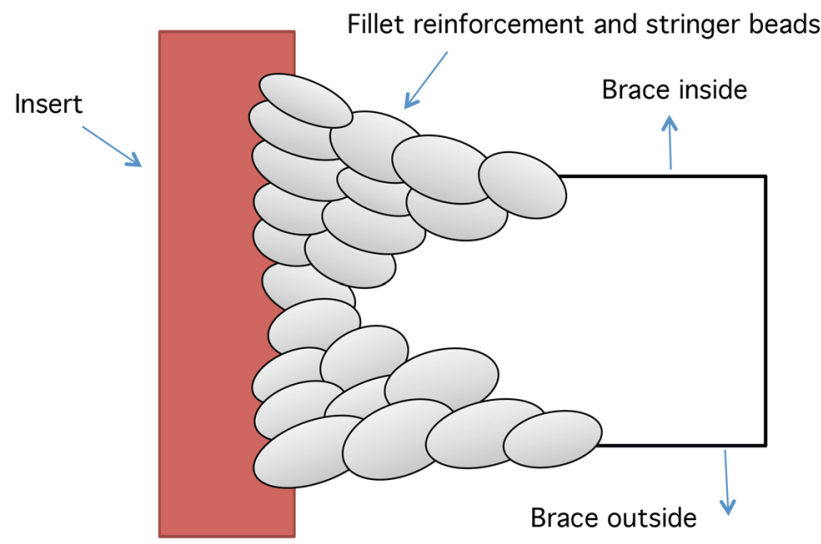

Fig. 5 Schematic representation of an insert full penetration butt weld

Fig. 3.1.9. of the accident report, these are the etched macrostructures at three different positions, positions P-85, P-94 and P-96, taken from the circumference/regions of hydrophone attachment remaining in place to the brace D6, reference pages 339 Fig. 3.1.5. and 345, Fig. 3.1.15 again from the accident report. On these macrographs, the vertical plates represent the brace material and the horizontal plate being the insert tube.

All three images possess features defining the inadequacy of the welding protocol, listed as;

- Inadequate penetration of the weld into the insert tube.

- Grossly different leg lengths on opposite sides of the brace plate.

- Evidence of excessive weaving of the electrode in an attempt to generate a sufficiency of fused weld material.
Figure 6: Classification of welds on the rig as stated by the accident report.

Class1:

--- mounting of nodes, and nodes between braces; and between braces and deck beams;

--- butt welds in the perimeter direction by prefabrication of braces made of rolled plates.

The welds of Class 1 , especially the nodal points were further divided into categories, $\mathrm{A}$ and $\mathrm{B}$, according to their importance. Of the node details special importance was attached to the corners of brackets.

Class 2 :

-- butt welds lengthwise by prefabrication of braces made of rolled plates;

--- fillet and butt welds connecting secondary parts of the structure and which are exposed to high tension.

Class 3 :

--- fillet and butt welds connecting secondary parts of the structure and which are exposed to low tension.

Fig. 6 Classification of welds on the rig as stated by the accident report

The weld instruction protocol stated as: the application of Manual Metal Arc (SMAW) using a ESAB OK 48.30 basic electrode of $5 \mathrm{~mm}$ diameter for this Class 3 joint.

The author identified the inadequate nature of the individual macrostructures as:

Position 85-imperfections

- Hydrogen induced cracks originating at the toe of the fillet and propagating into the insert plate material. The source of this hydrogen would be moisture on the surface of the joint, inadequate baking of the electrodes and poor control of arc length or a combination of these factors. The fusion and penetration characteristics of the weld deposits with the insert tube are low in comparison with the brace material, further identifying the application angle of the electrode which was predominantly perpendicular to the brace and only parallel to the insert. This feature of the application of the welding process by the welder resulted in unequal leg lengths of $12 \mathrm{~mm}$ on the brace and $8 \mathrm{~mm}$ on the insert tube.

Position 94-imperfections

- The welder continued the electrode weaving strategy and the same electrode application strategy generating unequal leg lengths and inadequate fusion profiles. On the left side weld weaving has induced the false nature of apparently four electrode passes where in reality it is from a single pass.

Position 96-imperfections 
Fig. 7 Macro-sections from around the remaining insert at pos. 85,94 and 96
NOU 1981: 11

-Alexander L. Klelland--ulykken

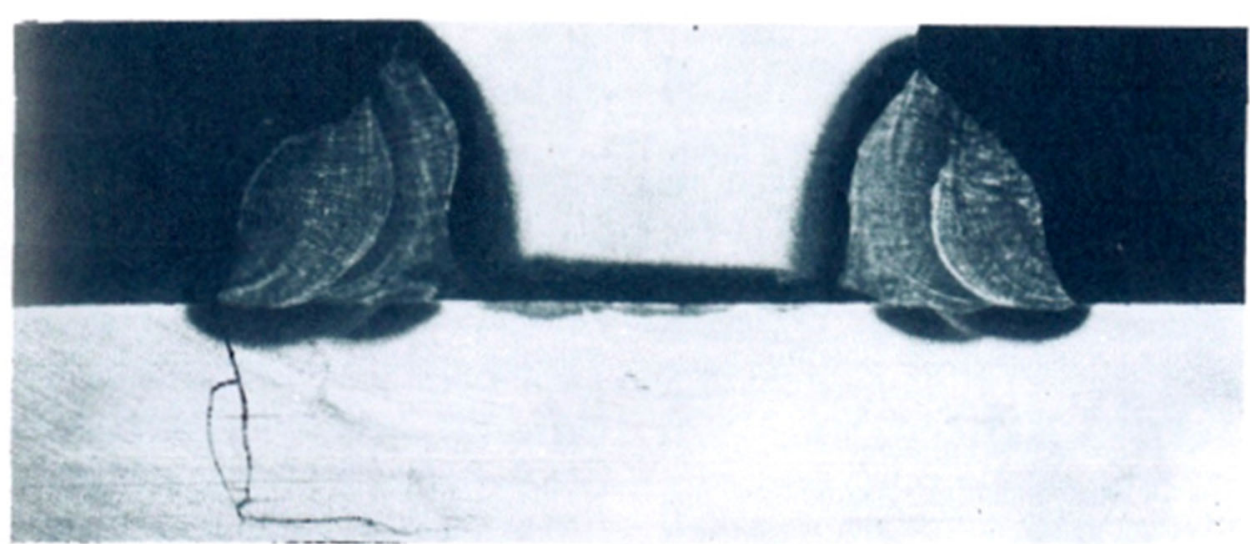

(a) Pos. 85
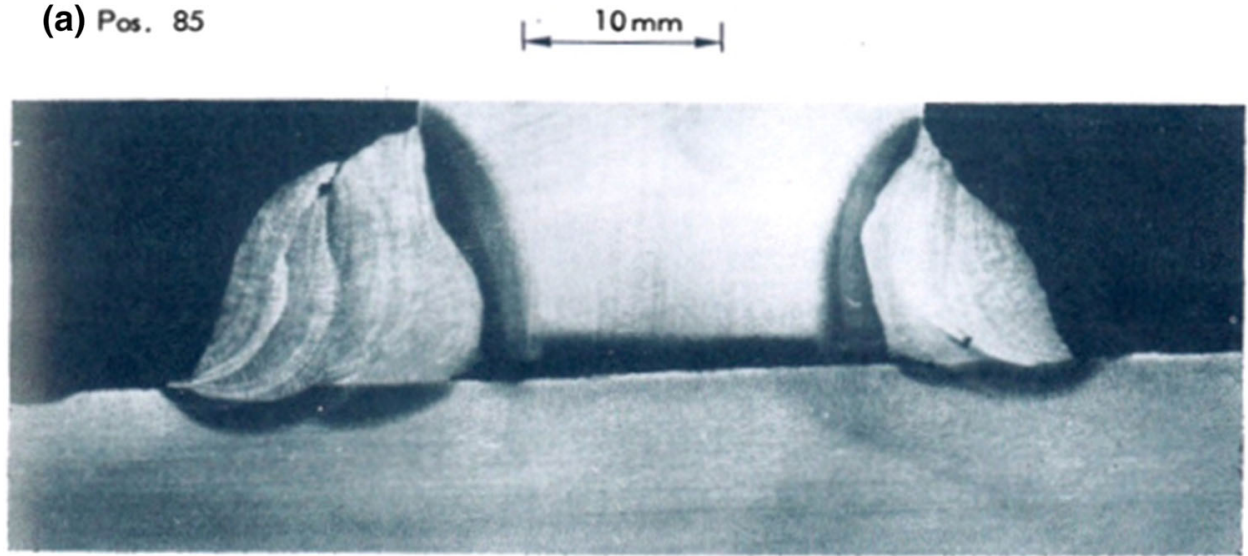

(b) Pos. 94
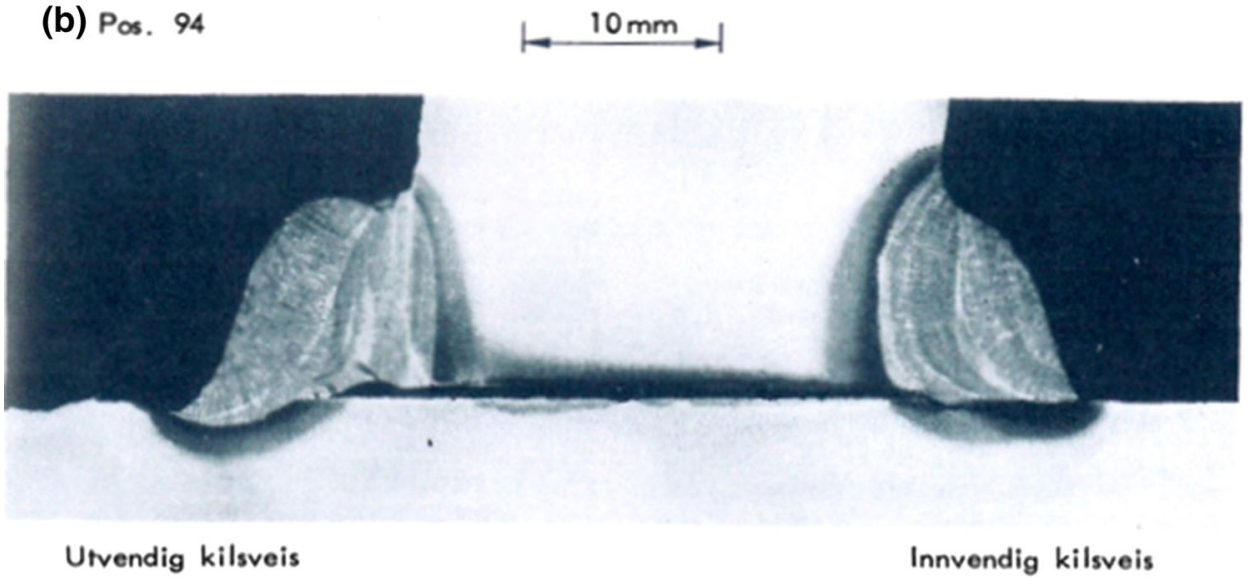

(c) Pos. 96

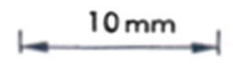

Innvendig kilsveis
- The application angle of the electrode is so exaggerated that there is complete lack of root fusion and significantly no horizontal insert sidewall fusion. The weld structure on the left side is due to two passes of the electrode compared with the right side deposit. By chance the fillet leg lengths are seen to be equal, creating the illusion that the joint is satisfactory. In reality the joint was poorly fused generating a potential point of failure.
All of the observed weld imperfections were a result of the manner in which the welder had applied the SMAW weld process. Clearly, this earliest evidence in the investigation confirms the failure to conduct a proper and acceptable weld procedure. The authors of the official report ignored the obvious deficiencies in weld production, seeking a mechanical property test study of the parent carbon steel plate. The mechanical testing of parent carbon 
steel served to confirm the as-supplied quality was compliant and in accordance with the steel specification.

At this stage of the accident investigation did the panel seek the advice of an experienced welding engineer? Such professional advice would have directed attention to the weldment imperfections of the welded insert joint, to the inappropriate application technique of the SMAW process, and would have questioned the selection of a Class 3 type classification for the joint. An unlikely feature of the insert weld, stated in the accident report, was the presence of paint layers on parts of the fracture surfaces. This evidence alone demonstrated the lack of even a simple and first visual inspection of the final welded joint required from the welder. Crack-like features are not permitted in all fabrication specifications; they are always classed as a defect, must be removed and weld repaired. Since there was no evidence presented to the panel, nor inferred, that a weld repair had been attempted, it must follow that a visual inspection was not applied.

Fatigue cracking usually emanates from a surface at points of highest stress and in fillet welds often at the toe sites. The toe profiles of the fillet in positions P-85, P-94 and P-96 are not blended correctly and could be preferred sites for crack initiation and propagation; the most likely site candidate, however, would be the hydrogen crack at P85 . The fact that the fatigue crack propagated from the fillet weld establishes the cyclic stress applied to the joint in service, due to imposed wave loading.

The author believes that there was a collective responsibility for the collapse of the platform, not stated in the final accident report. This conclusion follows from the question; "If the hydrophone insert had been welded correctly to deliver the required optimum weld properties, would the rig have failed in the way it did?" If the causes of failure of a welded structure are correctly identified, then the preventative actions would inhibit the mechanism of failure. In this scenario, the insert joint would be full strength, derived from being a full penetration butt type, correctly fused by a qualified welder, who would apply the SMAW process in accordance with a qualified and tested weld procedure. Under these criteria, the author considers it most unlikely that the insert weld joint would have initiated fatigue cracking, crack propagation and subsequent failure.

Let us compare the official explanation of the rig failure from the report ref. 1 with the view presented by the author of this article. The accident report concluded that the cause of failure occurred in the sequence below:

- Fatigue crack growth occurred from pre-existing cracks in the insert fillet welds between a hydrophone support and the brace D-6.
- Fatigue crack propagation part way around the circumference of the insert, then moving into the brace and around its circumference with brace failure by overload.

- The subsequent failure of the remaining five braces joining the column D-6 to the rig, by plastic collapse.

Further, the accident report [1] suggested that the carbon steel used in the brace manufacture possessed inadequate mechanical properties, but testing proved the materials met the supply specification requirements. It was also implied that the steels mechanical and physical properties played a role in the crack initiation and propagation mechanism. It did not recognize that fatigue failure has no regard for the tensile and ductility properties in all directions of rolled steel plate, since the stress concentration at the crack tip will override these properties.

Later in the accident report attention was focused upon the inadequate design of the platform, a factor considered as highly inappropriate in a situation in which pre-existing defects were not identified prior to service. These comments contained in the report concentrated upon the mechanism rather than the cause(s), of failure. The true cause of failure is found from an analysis of why fatigue cracks were so readily initiated in the fillet weld around the insert. The reality was that the fabrication classification for the hydrophone insert joint allowed the welders to substitute an imprudent weld practice, rather than using a qualified and fully tested weld procedure. The thick-walled joints elsewhere in the fabrication were strictly controlled under Class 1 and Class 2, but the design classification permitted the hydrophone insert to be welded out of control, i.e. Class 3, a situation both serious and dangerous. To designate any weld as of "no consequence" on such a structure is a deficient philosophy, and should not, under any circumstances, be adopted or permitted. Current specifications/classifications such as ISO 3834 suffer from the same flawed philosophy, part/section 4 being the culprit.

The role of the experienced welding engineer.

The author has attempted to demonstrate the effective role of the professional welding engineer, in all stages of large scale, welded fabrications. From the welding engineering viewpoint there was little or no consultation in this case study, with the mechanical engineering/design staff, evidenced by the poor choice of a small $6 \mathrm{~mm}$ throat thickness fillet to secure the hydrophone insert into a large diameter tubular brace. This choice would have been questioned immediately both from the potential stress levels imposed upon it in service and from the difficulty in meeting the necessary optimum mechanical properties in the weld bead/area. The accident report led to a demand for 
radical changes in all aspects of the mechanical design of this platform, with a total lack of focus on the true cause of collapse. A definitive welding engineering input in the construction of large-scale fabrications compliments the design strategy of the mechanical engineer. Clearly, any possible benefit that might accrue from a new engineering design would remain potentially at risk if the same deleterious welding protocols were repeated.

The bias in the accident report is shown by the conflicting clear evidence offered from an analysis of the weld characteristics and the history of the fracture progress in the fatigue induced collapse of the structure. The addition of a consulting welding engineer contributing an input to the accident report would have more persuasively identified the reality rather than post the responsibility upon a design fault.

It should be accepted here that any critical remarks in this article are meant to be taken from the point of view of constructive criticism. The evidence derived from this analysis should contribute to actions that could be taken to reduce the probability and the frequency of failures of this type. All of this information hopefully should be welcomed by anyone connected to the disaster and be regarded as an honorable objective to be achieved.

Acknowledgments Professor Barry G. Fookes, University of Central Florida, for a peer review of the article.

Open Access This article is distributed under the terms of the Creative Commons Attribution 4.0 International License (http:// creativecommons.org/licenses/by/4.0/), which permits unrestricted use, distribution, and reproduction in any medium, provided you give appropriate credit to the original author(s) and the source, provide a link to the Creative Commons license, and indicate if changes were made.

\section{Reference}

1. The Alexander L Kielland accident : report presented to the Ministry of Justice and Police, March 1981/from a commission appointed by Royal Decree of 28TH March 1980 [chairman : Thor Naesheim; Norway. Justis-og politidepartmentet]: Translation into English

Publisher's Note Springer Nature remains neutral with regard to jurisdictional claims in published maps and institutional affiliations. 